Original Article

\title{
N-Acetylcysteine's Renoprotective Effect in Cardiac Surgery: A Systematic Review and Meta-Analysis
}

\author{
Ying Kiat Tan, MBBS, ${ }^{1}$ HaiDong Luo, MD, PhD,${ }^{2}$ Giap Swee Kang, MBBS, FRCS, ${ }^{2}$ \\ Kristine LK Teoh, MBBS, FRCS, ${ }^{2}$ and Theo Kofidis, MD, FRCS ${ }^{2}$
}

\begin{abstract}
Objective: To examine N-acetylcysteine's (NAC's) renoprotective effect in adult cardiac surgery

Methods: PubMed, Ovid Medline, and Embase were searched for randomized controlled trials published between January 1990 and May 2021 that investigated the effect of NAC in preventing acute kidney injury (AKI) in patients undergoing cardiac surgery. The inclusion criterion was studies that assessed the effect of NAC in comparison to placebo by measuring the incidence of AKI.

Results: Overall meta-analytic estimates of all 10 included trials showed that NAC did not have a significant effect (odds ratio [OR]: $0.84,95 \%$ confidence interval [CI]: 0.64-1.10) on AKI. Further subgroup analysis did not show a significant benefit of NAC in preventing AKI.

Conclusion: This meta-analysis suggests that NAC does not have a significant effect in reducing the incidence of $\mathrm{AKI}$. However, there is notable heterogeneity among the included studies that could possibly account for the non-significant effect observed. It is worth noting that only one trial administered NAC high dosages perioperatively, and it is the only included trial to show a significant benefit in reducing the incidence of AKI (OR: 0.30, 95\% CI: 0.11-0.81). Further studies on this dosage and duration of administration should be conducted to best elucidate the effect of administering NAC.
\end{abstract}

Keywords: cardiac surgery, acute kidney injury, $\mathrm{N}$-acetylcysteine

${ }^{1}$ Department of Cardiac Surgery, Yong Loo Lin School of Medicine, Singapore, Singapore

${ }^{2}$ Department of Cardiac Thoracic \& Vascular Surgery, National University Heart Centre, Singapore, Singapore

Received: June 14, 2021; Accepted: August 4, 2021

Corresponding author: HaiDong Luo, MD, PhD. Department of Cardiac Thoracic \& Vascular Surgery, National University Heart Centre, 1E Kent Ridge Road, Singapore 119228, Singapore

Email: hai_dong_luo@nuhs.edu.sg

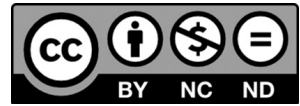

This work is licensed under a Creative Commons Attribution-NonCommercialNoDerivatives International License.

(C)2021 The Editorial Committee of Annals of Thoracic and Cardiovascular Surgery

\section{Introduction}

Description of condition

Acute kidney injury (AKI) is a common and serious complication after cardiac surgery. ${ }^{1)}$ Specifically, AKI is mostly caused by the usage of cardiopulmonary bypass (CPB) during coronary artery bypass grafting (CABG), and heart valve and aortic surgery. ${ }^{2)}$ The incidence of AKI is also dependent on the clinical characteristics of patients undergoing cardiac surgery, such as reduced left ventricular function, presence of congestive heart failure, or an elevated preoperative serum creatinine. AKI does not have a universal definition. The three most 
Tan $Y K$, et al.

popular consensus definitions are Acute Dialysis Quality Initiative's "RIFLE" criteria, the Acute Kidney Injury Network (AKIN) criteria, and the Kidney Disease Improving Global Outcomes criteria. ${ }^{3)}$ These criteria are based on percentage increase in serum creatine concentration over baseline, absolute increase in serum creatine concentration, or urine volume. ${ }^{4)}$ The pathogenesis of AKI after CPB is multifactorial, and is mostly due to hypoperfusion, reperfusion injury, activation of the systemic inflammatory response, and/or low cardiac output. ${ }^{5}$ Reperfusion will result in the formation of reactive oxygen species, resulting in injury to tissues. $\left.{ }^{6}\right)$ The activation of the systemic inflammatory response is mostly due to the exposure of blood to the extracorporeal $\mathrm{CPB}$ circuit, resulting in the activation of the immune system, which is also mediated by the generation of reactive oxygen species. This results in increased recruitment of neutrophils, macrophages, and lymphocytes into the renal parenchyma, leading to AKI.5)

\section{Description of intervention}

$\mathrm{N}$-acetylcysteine (NAC) is well recognized for its antioxidant and free-radical scavenging properties ${ }^{7)}$ in addition to acting as a vasodilator. ${ }^{8)}$ Its antioxidant properties allow it to inhibit ischemic cell death, and NAC, as a free-radical scavenger, attenuates the effect of increased reactive oxygen species caused by reperfusion. ${ }^{9}{ }^{9}$ Hence, theoretically, NAC is able to counteract several mechanisms of kidney injury during cardiac surgery, namely, the systemic inflammatory response, free-radical injury, and ischemia. ${ }^{9)}$

Many randomized controlled trials have been conducted to evaluate different measures to prevent AKI. These measures range from different pharmacological therapies, such as fenoldopam or pentoxifylline, to surgical techniques such as off-pump CPB to remote ischemic preconditioning. However, among the randomized controlled trials conducted on different preventive measures, the administration of NAC was the most promising measure with the greatest number of randomized controlled trials conducted on its usage. Hence, this review was conducted to evaluate the overall effect of NAC based on past randomized controlled trials.

\section{Material and Methods}

\section{Data sources, search strategy, and study selection}

Two reviewers (YKT and HDL) searched Medline (1990 to May 2021), Embase (1990 to May 2021), and
PubMed (1990 to May 2021) for randomized controlled studies that compared any form of usage of NAC to placebo in adult patients (age above 18) undergoing cardiac surgery. The following search terms were employed to source for eligible studies: NAC, N-acetylcysteine, Beta Blocker, Beta Antagonist, Cardiac surgery, Cardiac surgical procedures, Cardiac, Heart, Renal, Kidney, Acute Kidney Injury, and Acute Renal failure. There was no language restriction but only papers written in English were included.

\section{Inclusion criteria}

To be included, all studies had to assess the effect of NAC on the primary outcome of AKI after adult cardiac surgery. Only relevant randomized controlled trials conducted in the past 31 years (1990-2021) that were related to the search terms were included.

\section{Exclusion criteria}

Studies evaluating the effects of NAC in noncardiac surgical settings (such as contrast-induced nephropathy [CIN], endothelial function, and so on) and those that did not report the specified renal outcomes were excluded. Studies that lacked details on the timing and duration of administration of NAC or the exact amount of dosage administered were excluded. Studies of a small sample size, defined as having less than 10 patients in each arm of the randomized controlled trial, were also excluded.

\section{Data analysis}

Data were analyzed by Review Manager 5.4.1 (Cochrane, London, UK). Dichotomous data from individual studies were analyzed to calculate individual odds ratio $(\mathrm{OR})$ with $95 \%$ confidence interval $(\mathrm{CI})(\mathrm{p}<0.05)$ according to the inverse variance model. Statistical heterogeneity was analyzed using heterogeneity $\chi^{2}$ (Cochrane Q) statistic. Treatment effects were analyzed with the random-effects model.

\section{Results}

\section{Selection process}

Details of the flow of study identification are detailed in a Prisma chart shown in Fig. 1. Database searches yielded a total of 567 citations after removing duplicate citations. A total of 546 irrelevant citations were excluded based on abstract and title. After further assessment of the full text of the remaining citations, 11 were excluded due to not meeting the requirements of the selection criteria. These studies were excluded for the following 

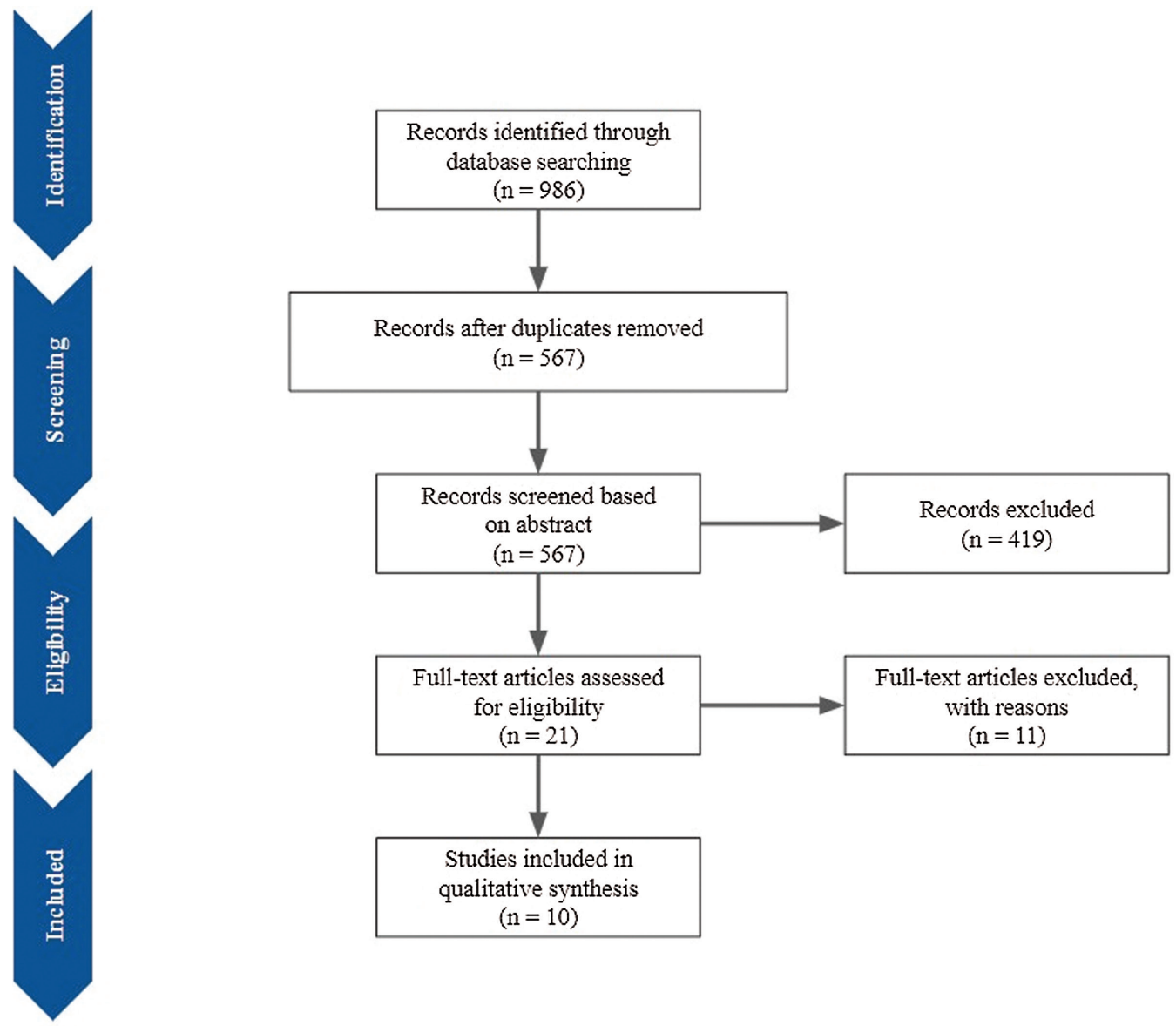

Fig. 1 Flow chart of study identification.

reasons: studies reported only on serum creatine levels $(\mathrm{n}=4)$, studies reported only on estimated glomerular filtration rate $(n=2)$, studies reported on outcomes other than the incidence of AKI $(n=2)$, studies reported on the usage of NAC in comparison to other drugs $(\mathrm{n}=2)$, and studies reported on the usage of NAC with other drugs $(\mathrm{n}=1)$. Our analysis finally identified 10 eligible studies comprising 1242 patients (623 NAC group, 619 control group). ${ }^{10-19)}$ Characteristics of the included studies are summarized in Supplemental Table 1 (All supplemental files are available Online).

\section{Risk of bias assessment}

The quality of the included studies was assessed by the Jadad scale. Supplemental Table 2 details the scores of each individual study. All included studies were assessed to have a low risk of bias.

\section{Characteristics of included studies}

There was no common definition of AKI among all 10 included studies. Seven out of 10 included studies either defined AKI or had their primary renal outcome defined as an increase in serum creatinine level greater than $0.5 \mathrm{mg} / \mathrm{dL}$ ( $44 \mu \mathrm{mol} / \mathrm{L}$ ) or a $25 \%$ increase from baseline. The remaining three studies defined AKI according to the AKIN criteria. In those studies, AKI was defined as an increase in serum creatinine more than or equal to $0.3 \mathrm{mg} / \mathrm{dl}$ from baseline, to $50 \%$ from baseline, or to an oliguria less than $0.5 \mathrm{ml} / \mathrm{kg} / \mathrm{h}$ for more than 6 hours, within the first 48 hours postoperative. In seven studies, patients underwent on-pump cardiac surgery, while patients in the other three studies underwent off-pump cardiac surgery. In five studies, NAC was administered perioperatively, while NAC was administered intraoperatively to postoperatively in the other five studies. Perioperative administration of NAC was defined as the administration of NAC during the preoperative, intraoperative, and postoperative phases. The patient study population also differs among the included studies, from patients with chronic kidney disease and different degrees of renal dysfunction to patients who had risk factors for postoperative renal dysfunction. 
Tan $Y K$, et al.

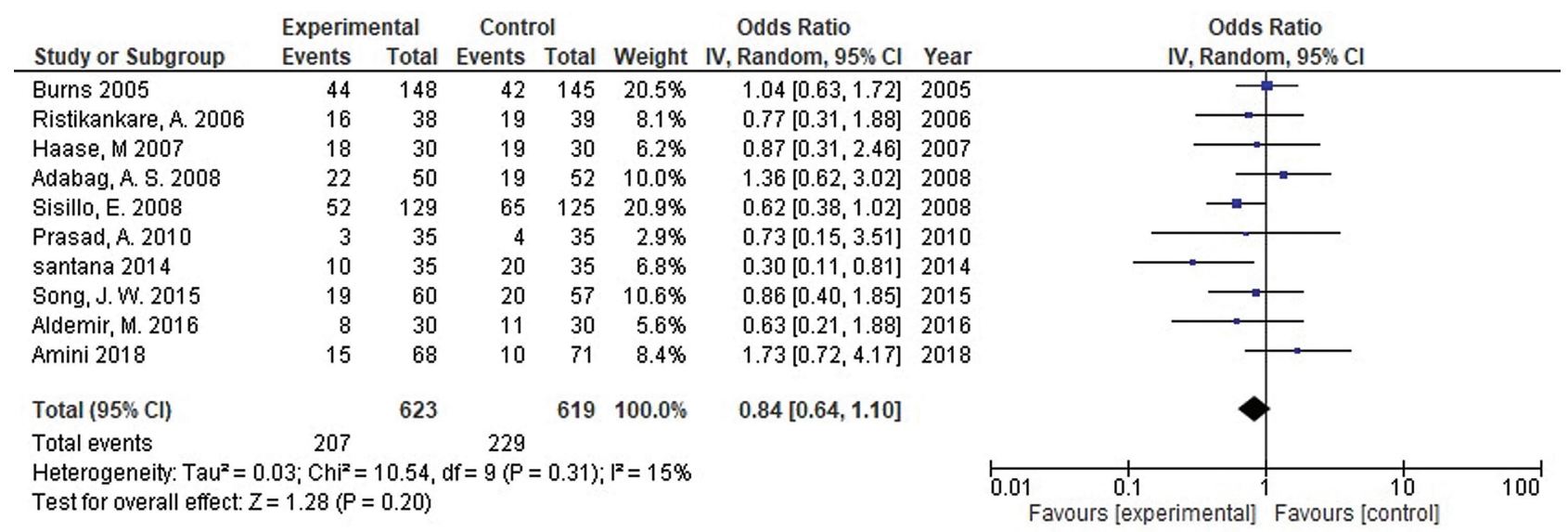

Fig. 2 Meta-analytic estimates of AKI comparing experiment (NAC) to control. AKI: acute kidney injury; CI: confidence interval; df: degrees of freedom; IV: intravenous; NAC: N-acetylcysteine

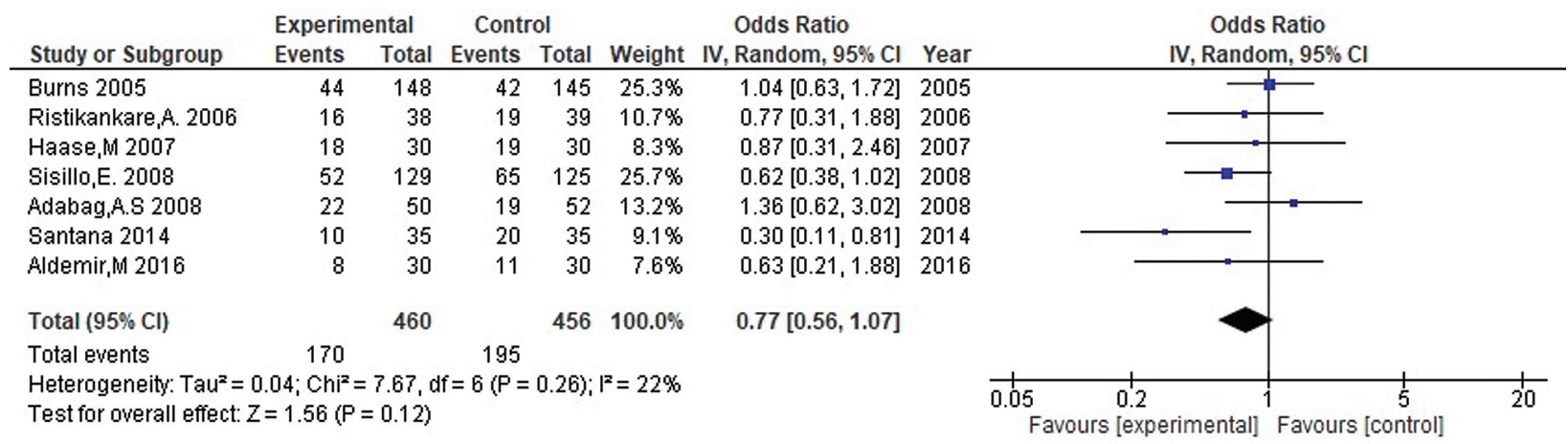

Fig. 3 Meta-analytic estimates of AKI comparing experiment (NAC) to control for on-pump surgery. AKI: acute kidney injury; CI: confidence interval; df: degrees of freedom; IV: intravenous; NAC: N-acetylcysteine

\section{Primary outcome measures}

Meta-analytic estimate showed a positive trend toward preventing AKI in patients treated with NAC. There was moderate statistical heterogeneity $\left(\mathrm{I}^{2}=31 \%\right)$ among the studies for the primary outcome. Overall results showed that the effect of NAC in preventing AKI after adult cardiac surgery was equivocal, as shown in Fig. 2 (OR: 0.84, 95\% CI: 0.64-1.10). As such, overall meta-analytic estimates showed that NAC did not have a significant effect; however, one study showed that NAC had significant benefits.

Stratified analysis of studies by the type of cardiac surgery did not show any significant benefit of NAC in reducing the incidence of AKI regardless of whether on-pump or off-pump surgery was conducted as shown in Fig. 3 (OR: 0.77, 95\% CI: 0.56-1.07) and Fig. 4 (OR: 1.10, 95\% CI: 0.64-1.89), respectively.

Stratified analysis of studies by the degree of preexisting kidney impairment did not show any significant benefit of NAC in reducing the incidence of AKI regardless of whether NAC was administered in patients with mild to moderate kidney impairment or chronic kidney disease. This is shown in Fig. 5 (OR: 1.01, 95\% CI: 0.73-1.41) and Supplemental Fig. 1 (OR: 0.66, 95\% CI: 0.32-1.36), respectively.

Perioperative usage of NAC was examined in five studies. Intraoperative to postoperative usage of NAC was examined in five studies where NAC was administered after the induction of anesthesia and administered for 12-24 hours postoperatively. Meta-analytic estimates of perioperative usage and intraoperative to postoperative usage are detailed in Supplemental Figs. 2 and 3.

As the format of the dosages of NAC reported varied greatly among all 10 included studies, the format of the dosages was stratified into Protocol A and Protocol B. Protocol A was administered in five studies, where generally, the dosage administered was $150 \mathrm{mg} / \mathrm{kg}$ for 15 minutes, $50 \mathrm{mg} / \mathrm{kg}$ for the next 4 hours, and $100 \mathrm{mg} /$ $\mathrm{kg}$ for the next 16 to 20 hours. Protocol B was administered in five studies, where each dose was administered 


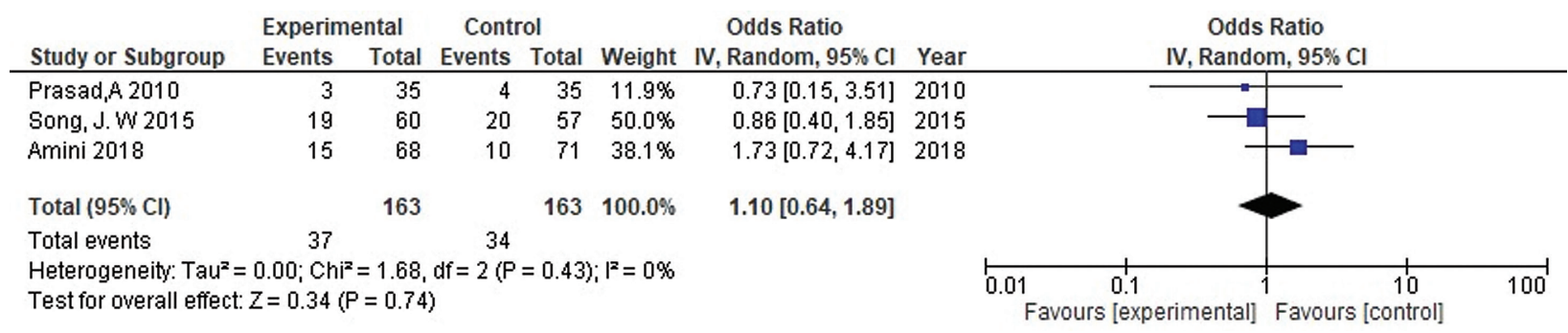

Fig. 4 Meta-analytic estimates of AKI comparing experiment (NAC) to control for off-pump surgery. AKI: acute kidney injury; CI: confidence interval; df: degrees of freedom; IV: intravenous; NAC: N-acetylcysteine

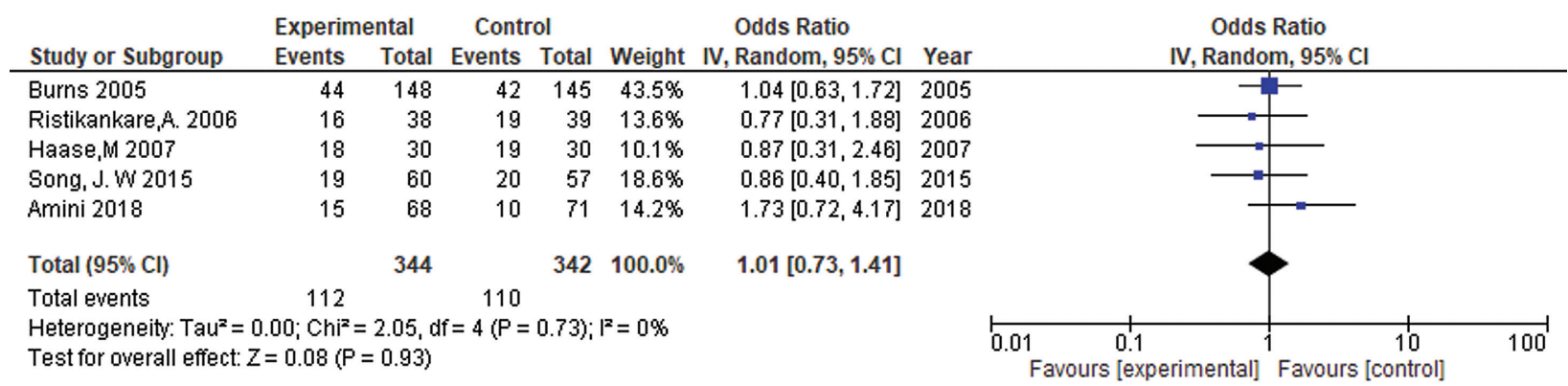

Fig. 5 Meta-analytic estimates of AKI comparing experiment (NAC) to control for patients with mild to moderate kidney impairment. AKI: acute kidney injury; CI: confidence interval; df: degrees of freedom; IV: intravenous; NAC: N-acetylcysteine

at $600 \mathrm{mg}$, with the number of doses varying across the five studies. It is worth noting that Protocol A involved a much higher dosage of NAC administered compared to Protocol B. These characteristics of the studies are summarized in Supplemental Table 3. Meta-analytic estimates of Protocol A and Protocol B are detailed in Supplemental Figs. 4 and 5.

\section{Secondary outcomes}

Three secondary outcomes, in-hospital mortality, requirement for renal replacement therapy, and length of stay (LOS) in the intensive care unit (ICU), were also assessed. Regarding in-hospital mortality, a total of 1055 patients in eight studies were observed. Meta-analytic estimates for in-hospital mortality showed that there was no statistically significant difference between patients treated with NAC and without, as shown in Supplemental Fig. 6 (OR: 0.85, 95\% CI: 0.43-1.68).

Regarding the requirement for renal replacement therapy, a total of 686 patients in four studies were assessed. Meta-analytic estimates did not show any significant benefit of NAC in reducing the requirement for renal replacement therapy as shown in Supplemental Fig. 7 (OR: 1.05, 95\% CI: 0.38-2.92).

Seven studies (1042 patients) reported LOS in the ICU. All studies reported the exact LOS in the ICU except one study that reported the number of patients who had an ICU stay longer than 4 days. Three studies reported the mean LOS and standard deviation. Two studies reported the median LOS and interquartile range. One study reported the median LOS and total range. For meta-analytic estimates to be conducted, a normal distribution had to be assumed for the three studies that reported the median LOS. Thus, the median was approximated to be the mean, and the standard deviation was approximated by dividing the interquartile range by 1.35 and the total range by 4 , as advised by Cochrane. ${ }^{20)}$ Meta-analytic estimates of studies that reported the mean and standard deviation, and studies with approximated mean and standard deviation were conducted separately. Both meta-analytic estimates did not show any benefit of NAC in reducing the LOS in the ICU as shown in Supplemental Fig. 8 (mean difference: 0.27, 95\% CI: -2.25 to 2.79) and Supplemental Fig. 9 (mean difference: $0.00,-0.27$ to 0.27 ).

\section{Publication bias}

Publication bias was assessed by Review Manager V5.3 (Cochrane), and a funnel plot for the analysis of complication rate is shown in Supplemental Fig. 10. The symmetrical funnel plot indicates a low risk of publication bias. 
Tan $Y K$, et al.

\section{Discussion}

It is intriguing that despite NAC's well-documented effect as an antioxidant, vasodilator, and free-radical scavenger, overall data analysis was inconclusive in showing that NAC could provide significant renal protection in major cardiac surgery. In addition, NAC has been shown to be effective in preventing radiocontrast nephropathy ${ }^{21)}$ and protecting renal function after $\mathrm{CBP},{ }^{22)}$ possibly by inhibiting transforming growth factor beta 1.23) Animal models have also shown that administration of NAC reduces total, cortical, and medullary vascular resistance by $7 \%$ to $10 \%$, improving renal function, ${ }^{24)}$ which further suggests that NAC could confer protection against AKI.

It is likely that the main reason for the controversial effect of NAC is due to the heterogeneity of the clinical trial included in this meta-analysis. Noticeably, the administration of NAC (dosage, timing, and overall duration), type of cardiac surgery, inclusion criteria of patients, and definition of AKI differed across the included studies.

\section{Administration of NAC}

There were four studies that administered NAC perioperatively via Protocol B and four studies that administered NAC intraoperatively to postoperatively via Protocol A. There was one study that administered NAC perioperatively via Protocol A and one study that administered NAC intraoperatively to postoperatively via Protocol B. Based on the stratified meta-analysis according to dosage, it is worth noting that the effect of NAC in preventing renal failure was more pronounced in studies where it was administered at a much higher dosage (Protocol A). All five studies that reported higher doses of NAC ${ }^{11-15)}$ showed a trend pointing toward the reno-protective effect of NAC, with an OR of less than 1. This is further supported when meta-analytic estimates of NAC administered at high dosages/Protocol A (Supplemental Fig. 4) were compared to that of low dosages/Protocol B (Supplemental Fig. 5). The OR of all studies that administered high dosages of NAC was at 0.66 (95\% CI: 0.44-1.01), while that of studies that administered low dosages of NAC was at 0.98 (95\% CI: 0.67-1.42). Second, NAC's effect on preventing against renal failure was more pronounced in studies where it was administered perioperatively. In two studies conducted by Prasad et al. ${ }^{10)}$ and Santana-Santos et al., ${ }^{11}$ perioperative usage of NAC resulted in a decrease in the incidence of renal failure, with an OR of 0.73 and 0.30 , respectively. It is also worth noting that the study conducted by Santana-Santos and associates showed a significant benefit in perioperative usage of NAC (OR: 0.30, 95\% CI: 0.11-0.81). However, when meta-analytic estimates of all perioperative usages of NAC (Supplemental Fig. 2) were compared to those of intraoperative to postoperative usages of NAC (Supplemental Fig. 3), the trend showed that intraoperative to postoperative usages of NAC had a more pronounced effect (OR: 0.71, 95\% CI: 0.50-0.99) as compared to the perioperative usage of NAC (OR: 0.95, 95\% CI: 0.55-1.63). We speculate that this could be due to the fact that a low dosage was administered in three of the five studies analyzed in the meta-analysis on the perioperative usage of NAC, skewing the results in favor of intraoperative to postoperative usage.

\section{Type of cardiac surgery/inclusion criteria of patients}

Other possible explanations for NAC's inconsistent effect on renal function could be due to differences in off-pump and on-pump CABG. The fact that the systemic inflammatory response is more likely to be activated when exposed to extracorporeal CPB circuit was only found in on-pump CABG. The multifactorial pathophysiology of AKI after cardiac surgery could suggest that other mechanisms unaffected by NAC could have resulted in renal failure despite administration of NAC, such as low cardiac output and decreased perfusion pressure. Lastly, for studies which focused on patients with chronic kidney disease or moderate renal insufficiency undergoing cardiac surgery, the effect of NAC may be insufficient in protecting renal function since renal function was already compromised prior to cardiac surgery. Although stratified analysis by the type of cardiac surgery or inclusion criteria of patients failed to show a significant benefit, this is likely due to the differing dosage and administration of NAC across studies.

\section{Definition of AKI}

The definition of AKI varied across all studies. While the majority of studies shared a common parameter of a $25 \%$ increase in serum creatinine over baseline, it is worth noting that most of the studies had varying alternative criteria of defining $\mathrm{AKI}$, which was usually via different absolute increases in serum creatinine levels. 
This could possibly result in the incidence of AKI across different studies being inconsistent.

\section{Limitations}

The main limitation of this study is the heterogeneity in study design across the randomized controlled trials, from study population, inclusion criteria for patients, type of surgery, and definition of AKI to administration of NAC. As a result, the exact effect of NAC cannot be elucidated as there are too many confounding factors at play, despite our best efforts to stratify analysis according to studies with the similar dosages (Protocol A vs Protocol B) or type of cardiac surgery (on-pump vs off-pump).

However, we hypothesize that NAC's renoprotective effect is best elucidated when administered at high dosages or Protocol A perioperatively. This is supported by the fact that among all 10 studies, only one study reported the administration of high doses of NAC perioperatively, ${ }^{11)}$ and that study was the sole study to show a significant benefit (OR: $0.30,95 \%$ CI: 0.11-0.81). This study was accorded a greater weightage compared to other studies due to it having a significant number of patients and being of better scientific design, as a double-blind randomized controlled trial. In addition, the existing literature also lends weight to this hypothesis, with studies showing that high doses of NAC have a renoprotective effect in preventing the development of CIN. ${ }^{25}$ ) As AKI after cardiac surgery shares a similar pathophysiology to CIN, it is likely that high doses of NAC administered perioperatively would have the best effect in reducing the incidence of AKI.

It is also worth mentioning that one of the studies excluded $^{26)}$ also showed a significant reduction in the incidence of AKI when carvedilol, a beta-blocker, was administered with NAC (OR: 0.42, 95\% CI: 0.23, 0.79). This study was excluded as it lacked a study arm where only NAC was administered. However, it is likely that the significant effect shown in this study is due to NAC, since there was a decreased incidence of AKI in patients who were administered both NAC and carvedilol as compared to patients who were only administered carvedilol. The theoretical rationale for the administration of both drugs is that since both drugs have antioxidant and anti-inflammatory effects, their combination might have a better effect in reducing the incidence of AKI. More importantly, NAC was administered perioperatively at high dosages, which further suggests that the NAC's effect on preventing renal failure is maximized when administered perioperatively at high dosages.

\section{Relation to other studies}

A previous meta-analysis on the effect of NAC in reducing the incidence of $\mathrm{AKI}$ after cardiac surgery shares similar limitations with this meta-analysis, ${ }^{27)}$ resulting in similar conclusion showing a non-significant effect of NAC. Nevertheless, we believe that this meta-analysis is unique in proposing that the renoprotective effect of NAC may be best elucidated when high doses of NAC is administered perioperatively. Further randomized controlled trials should thus be conducted to examine the effects of administering high dosages of NAC perioperatively. This would better elucidate the benefits of administering NAC in order to maximize patient outcomes.

\section{Conclusion}

Meta-analysis of the effects of NAC in preventing AKI after cardiac surgery is hard to interpret due to different dosages and different treatment time periods across different studies. However, further analysis shows that perioperative high dosages of NAC could have a significant protective effect in preventing renal failure after adult cardiac surgery. Further randomized controlled trials should be conducted to examine the effects of administering high dosages of NAC perioperatively, possibly with beta-blocker agents administered as well. This would better elucidate the benefits of administering NAC in order to maximize patient outcomes.

\section{Disclosure Statement}

The authors have no conflicts of interests to disclose.

\section{References}

1) Lee $\mathrm{SH}$, Kim SJ, Kim HJ, et al. Acute kidney injury following cardiopulmonary bypass in children-risk factors and outcomes. Circ J 2017; 81: 1522-7.

2) Schopka S, Diez C, Camboni D, et al. Impact of cardiopulmonary bypass on acute kidney injury following coronary artery bypass grafting: a matched pair analysis. J Cardiothorac Surg 2014; 9: 20.

3) Waikar SS, Bonventre JV. Creatinine kinetics and the definition of acute kidney injury. J Am Soc Nephrol 2009; 20: 672-9. 
Tan $Y K$, et al.

4) Kumar AB, Suneja M. Response: role of true cardiopulmonary bypass time and acute kidney injury after cardiopulmonary bypass. J Cardiothorac Vasc Anesth 2012; 26: E19-20.

5) Nadim MK, Forni LG, Bihorac A, et al. Cardiac and vascular surgery-associated acute kidney injury: The 20th International Consensus Conference of the ADQI (Acute Disease Quality Initiative) Group. J Am Heart Assoc 2018; 7: e008834.

6) Sinning C, Westermann D, Clemmensen P. Oxidative stress in ischemia and reperfusion: current concepts, novel ideas and future perspectives. Biomark Med 2017; 11: 11031-40.

7) Ates B, Abraham L, Ercal N. Antioxidant and free radical scavenging properties of $\mathrm{N}$-acetylcysteine amide (NACA) and comparison with $\mathrm{N}$-acetylcysteine (NAC). Free Radic Res 2008; 42: 372-7.

8) Nigwekar SU, Kandula P. N-Acetylcysteine in cardiovascular-surgery-associated renal failure: a metaanalysis. Ann Thorac Surg 2009; 87: 139-47.

9) Savluk OF, Guzelmeric F, Yavuz Y, et al. N-acetylcysteine versus dopamine to prevent acute kidney injury after cardiac surgery in patients with preexisting moderate renal insufficiency. Braz J Cardiovasc Surg 2017; 32: 8-14.

10) Prasad A, Banakal S, Muralidhar K. N-acetylcysteine does not prevent renal dysfunction after off-pump coronary artery bypass surgery. Eur J Anaesthesiol 2010; 27: 973-7.

11) Santana-Santos E, Gowdak LH, Gaiotto FA, et al. High dose of $\mathrm{N}$-acetylcystein prevents acute kidney injury in chronic kidney disease patients undergoing myocardial revascularization. Ann Thorac Surg 2014; 97: 1617-23.

12) Ristikankare A, Kuitunen T, Kuitunen A, et al. Lack of renoprotective effect of i.v. $\mathrm{N}$-acetylcysteine in patients with chronic renal failure undergoing cardiac surgery. Br J Anaesth 2006; 97: 611-6.

13) Haase M, Haase-Fielitz A, Bagshaw SM, et al. Phase II, randomized, controlled trial of high-dose Nacetylcysteine in high-risk cardiac surgery patients. Crit Care Med 2007; 35: 1324-31.

14) Song JW, Shim JK, Soh S, et al. Double-blinded, randomized controlled trial of $\mathrm{N}$-acetylcysteine for prevention of acute kidney injury in high risk patients undergoing off-pump coronary artery bypass. Nephrology (Carlton) 2015; 20: 96-102.

15) Aldemir M, Koca HB, Baki ED, et al. Effects of $\mathrm{N}$-acetyl cysteine on renal functions evaluated by blood neutrophil gelatinase-associated lipocalin levels in geriatric patients undergoing coronary artery bypass grafting. Anatol J Cardiol 2016; 16: 504-11.

16) Burns K, Chu M, Novick R. Perioperative Nacetylcysteine to prevent renal dysfunction in high-risk patients undergoing CABG surgery: a randomized controlled trial. JAMA 2005; 294: 342-50.

17) Adabag AS, Ishani A, Koneswaran S, et al. Utility of $\mathrm{N}$-acetylcysteine to prevent acute kidney injury after cardiac surgery: a randomized controlled trial. Am Heart J 2008; 155: 1143-9.

18) Sisillo E, Ceriani R, Bortone F, et al. N-acetylcysteine for prevention of acute renal failure in patients with chronic renal insufficiency undergoing cardiac surgery: a prospective, randomized, clinical trial. Crit Care Med 2008; 36: 81-6.

19) Amini S, Robabi HN, Tashnizi MA, et al. Selenium, vitamin $\mathrm{C}$ and $\mathrm{N}$-acetylcysteine do not reduce the risk of acute kidney injury after off-pump CABG: a randomized clinical trial. Braz J Cardiovasc Surg 2018; 33: $129-34$.

20) Cochrane handbook for systematic reviews of interventions. Cochrane Training. Trusted evidence. Informed decision. Better health. n.d. https://training. cochrane.org/handbook/current

21) Xu R, Tao A, Bai Y, et al. Effectiveness of Nacetylcysteine for the prevention of contrast-induced nephropathy: a systematic review and meta-analysis of randomized controlled trials. J Am Heart Assoc 2016; 5: e003968.

22) Sucu N, Cinel I, Unlu A, et al. N-acetylcysteine for preventing pump-induced oxidoinflammatory response during cardiopulmonary bypass. Surg Today 2004; 34: 237-42.

23) Qu X, Li Q, Wang X, et al. N-acetylcysteine attenuates cardiopulmonary bypass-induced lung injury in dogs. J Cardiothorac Surg 2013; 8: 107.

24) Fishbane S. N-acetylcysteine in the prevention of contrast-induced nephropathy. Clin J Am Soc Nephrol 2008; 3: 281-7.

25) Jo SH. N-acetylcysteine for prevention of contrastinduced nephropathy: a narrative review. Korean Circ J 2011; 41: 695-702.

26) Ozaydin M, Peker T, Akcay S, et al. Addition of $\mathrm{N}$-acetyl cysteine to carvedilol decreases the incidence of acute renal injury after cardiac surgery. Clin Cardiol 2014; 37: 108-14.

27) Pereira JEG, El Dib R, Braz LG, et al. N-acetylcysteine use among patients undergoing cardiac surgery: a systematic review and meta-analysis of randomized trials. PLoS One 2019; 14: e0213862. 